Thorax, 1978, 33, 31-42

\title{
Evagination of smooth muscle cells in the hypoxic pulmonary trunk
}

\author{
PAUL SMITH, DONALD HEATH, AND FABRIZIO PADULA \\ From the Department of Pathology, University of Liverpool
}

Smith, P., Heath, D., and Padula, F. (1978). Thorax, 33, 31-42. Evagination of smooth muscle cells in the hypoxic pulmonary trunk. Six female Wistar albino rats were exposed to the hypoxia of a simulated altitude of $5500 \mathrm{~m}$, three for a period of one week and three for a month. They developed ultrastructural changes in the pulmonary trunk consisting of evaginations of muscle cells of its media through gaps in the internal elastic lamina to press into the underlying endothelial cells. Such evaginations were usually devoid of myofilaments and organelles. Some appeared so electron-lucent as to be unrecognisable as muscle apart from the unequivocal connection with the parent smooth muscle cells. Elsewhere we have demonstrated that muscular evaginations in normal pulmonary blood vessels are an artefact brought about by collapse of lung tissue and that they can be avoided by distending the lung. Hence in the present investigation, in which the pulmonary trunk was fixed in distension, the evaginations are interpreted as indicating contraction of the muscle cells able to overcome the distending force. We interpret them as evidence of constriction of muscle cells in the media of the pulmonary trunk in response to hypoxia.

We have recently studied the ultrastructure of the smallest pulmonary arteries and the pulmonary arterioles in rats acutely exposed to severe hypoxia (Smith and Heath, 1977). We thought it would be of interest to see if the same hypoxic stimulus brought about electron microscopic changes in the smooth muscle cells situated in the other extremity of the pulmonary circulation, namely, in the pulmonary trunk. Our findings are described in this paper.

\section{Material and methods}

Ten female Wistar albino rats, weighing 250 $300 \mathrm{~g}$, were divided into groups of six test animals and four controls. The animals were housed in individual cages and given free access to food and water. The test rats were placed in a decompression chamber, and the pressure was reduced to $500 \mathrm{mmHg}$ for the first 24 hours to permit them to acclimatise. Thereafter the pressure was maintained at $380 \mathrm{mmHg}$, simulating an altitude of $5500 \mathrm{~m}$. The control animals were kept in the same room at normal sea-level atmospheric pressure. The pressure in the hypobaric chamber was briefly returned to normal for 20 minutes twice a week in order that the cages could be cleaned and the food and water replenished. After one week three test rats were removed from the chamber and killed while the remaining three remained in the chamber for a total of four weeks. Two control rats were also killed on the first and fourth weeks.

Immediately after removal from the chamber the animals were anaesthetised with ether vapour and the thorax and abdomen were opened by a mid-line incision. The carotid arteries and superior venae cavae were ligated. A small incision was made in the inferior vena cava between the origins of the renal veins, and a polythene cannula was passed into the vessel towards the heart. The cannula was tied firmly into place. The abdominal aorta was also similarly cannulated close to its bifurcation. The cannula within the inferior vena cava was connected to a flask containing heparinised saline, which entered the inferior vena cava at a pressure of $20 \mathrm{cmH}_{2} \mathrm{O}$. This flushed out the inferior vena cava, pulmonary arteries, and pulmonary veins, the blood and surplus saline being collected via the aortic cannula. When a threeway stopcock was turned the saline was replaced by chilled, buffered glutaraldehyde. After this had 
filled the pulmonary vasculature and had started to flow from the aorta, the aortic cannula was clamped to reduce the flow of fixative to approximately $1 \mathrm{ml} / \mathrm{min}$. By this means the full hydrostatic pressure of $20 \mathrm{cmH}_{2} \mathrm{O}$ was applied to the pulmonary trunk in order to fix it as close to its dimensions in vivo as possible. Fixation was aided by pouring glutaraldehyde onto the outside surface of the pulmonary trunk. It was left to fix for two hours.

The thoracic organs were then removed, and the pulmonary trunk was carefully dissected free and stored overnight in glutaraldehyde at $4^{\circ} \mathrm{C}$. Small arcs of pulmonary trunk were trimmed off the vessel, post-fixed for one hour in $1 \%$ osmium tetroxide, stained with uranyl acetate, and embedded in Araldite. Semithin sections, $1 \mu \mathrm{m}$ in thickness, were stained with toluidine blue for the selection of a suitable field. Blocks were selected in which the elastic laminae were thin and clearly defined and had not been sectioned obliquely. Fine sections were cut with an LKB Ultrotome III, stained with lead citrate, and examined with an AEI EM 6B electron microscope. An average of five blocks was examined from each rat in both the test and control groups.

\section{Results}

CONTROL RATS

The ultrastructure of the pulmonary trunk com- $\vec{\circ}$ prised three clearly defined concentric zones. The intima was lined by a thin layer of endothelium. The endothelial cells contained Weibel-Palade bodies but lacked the large discoid bodies which are so much a feature of the smaller pulmonary blood vessels in rats (Smith and Heath, 1977). Beneath the endothelium was a broad zone of $\dot{\omega}$ subendothelial connective tissue (Fig. 1). This $\circ$ consisted of a haphazard mixture of collagen fibrils, ground substance, and small 'islands' of T1 cytoplasm derived from inward extensions of endothelial cells. This subendothelial connective tissue layer was much thicker than that in muscular pulmonary arteries, in which there was merely a thin basement membrane.

Immediately subjacent to the intima was the electron-lucent internal elastic lamina (Fig. 1).

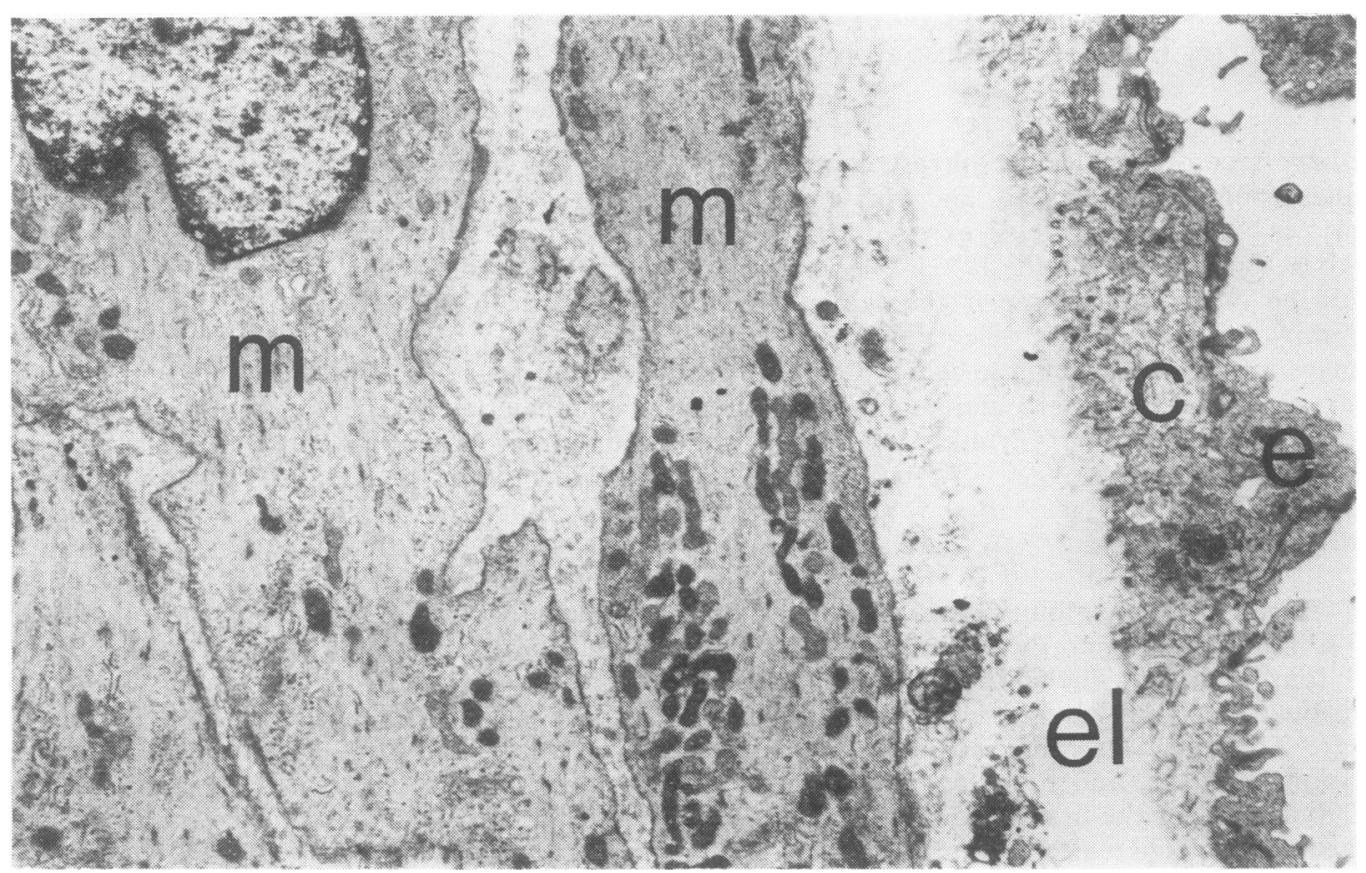

Fig. 1 Pulmonary trunk of a control rat showing the typical ultrastructure of this vessel. The lumen is lined by a thin layer of endothelial cells $(e)$, beneath which is a broad layer of subendothelial connective tissue (c). The internal elastic lamina (el) separates the intima from the media, which consists of numerous smooth muscle cells $(m)$ containing mitochondria and myofilaments. There is a wide zone of connective tissue between adjacent muscle cells. The medial elastic laminae are not illustrated. Electron micrograph $\times 7500$. 
Unlike the muscular pulmonary arteries, the internal elastic lamina in the pulmonary trunk was discontinuous (Fig. 2). These short gaps in the lamina probably represent spaces between the adjacent elastin fibres which form the woven network of each elastic lamina in the pulmonary trunk (Smith, 1977).

The media of the pulmonary trunk consisted of alternating bands of smooth muscle separated by elastic laminae. The muscle bands were several cells in thickness and the medial elastic laminae were also discontinuous. Thin bands of collagen and elastin interconnected adjacent elastic laminae. The smooth muscle cells were widely separated by a loose mixture of collagen and ground substance (Fig. 1). Where there were gaps in the internal elastic lamina the medial connective tissue extended through the gap and fused with the subendothelial connective tissue. Occasionally, however, extrusions of medial smooth muscle had herniated through these gaps to adopt a position in the intima (Fig. 2). Such extrusions in the distended pulmonary trunk of the control animals were, however, small and uncommon.

\section{TEST RATS}

Exposed to one week of hypoxia

The most striking feature of the pulmonary trunk after one week of hypoxia was the presence in the intima of numerous islands of cytoplasm (Fig. 3). These were embedded within the subendothelial connective tissue, often close to a gap in the internal elastic lamina. Their cytoplasmic density was often similar to that of smooth muscle (Fig. 3), but, unlike muscle, they did not contain myofilaments or mitochondria, and their endoplasmic reticulum was disrupted (Figs 3-5). At first the nature of these cytoplasmic islands was unclear, but it became apparent that they were evaginations of smooth muscle from the media, similar to those in the controls but much more widespread. Some of the cytoplasmic islands had been sectioned fortuitously so that their connection with parent muscle cells in the media through gaps in the elastic lamina could be traced (Fig. 4). The muscular nature of the parent cells was confirmed by their containing myofilaments and dense attachment points (Fig. 5).

In some areas the muscular evaginations were pale and devoid of organelles (Fig. 6). These

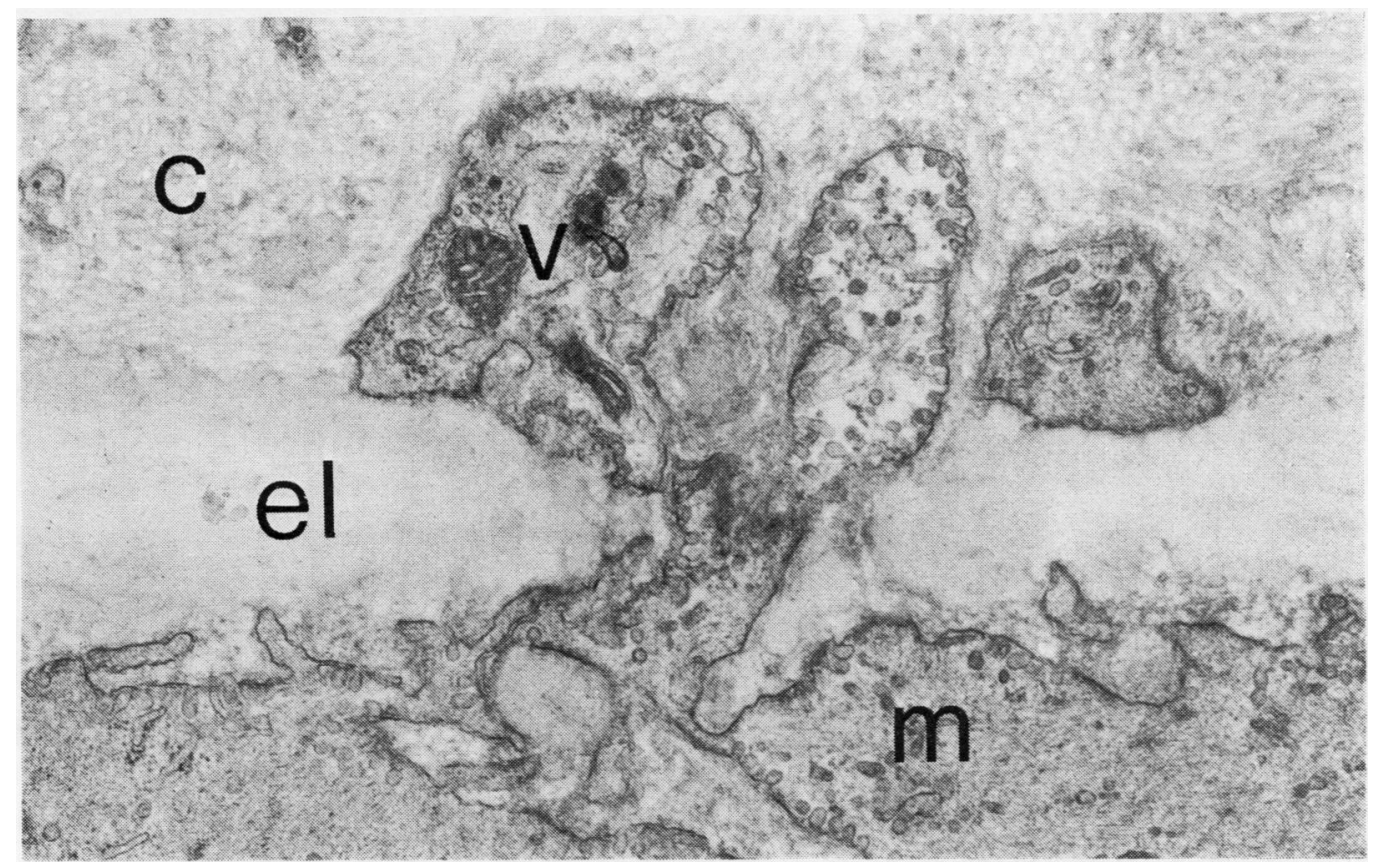

Fig. 2 Control pulmonary trunk. A smooth muscle cell $(m)$ has extended through a gap in the internal elastic lamina (el) to produce muscular evaginations $(v)$ in the subendothelial connective tissue $(c)$. Electron micrograph $\times 25000$. 


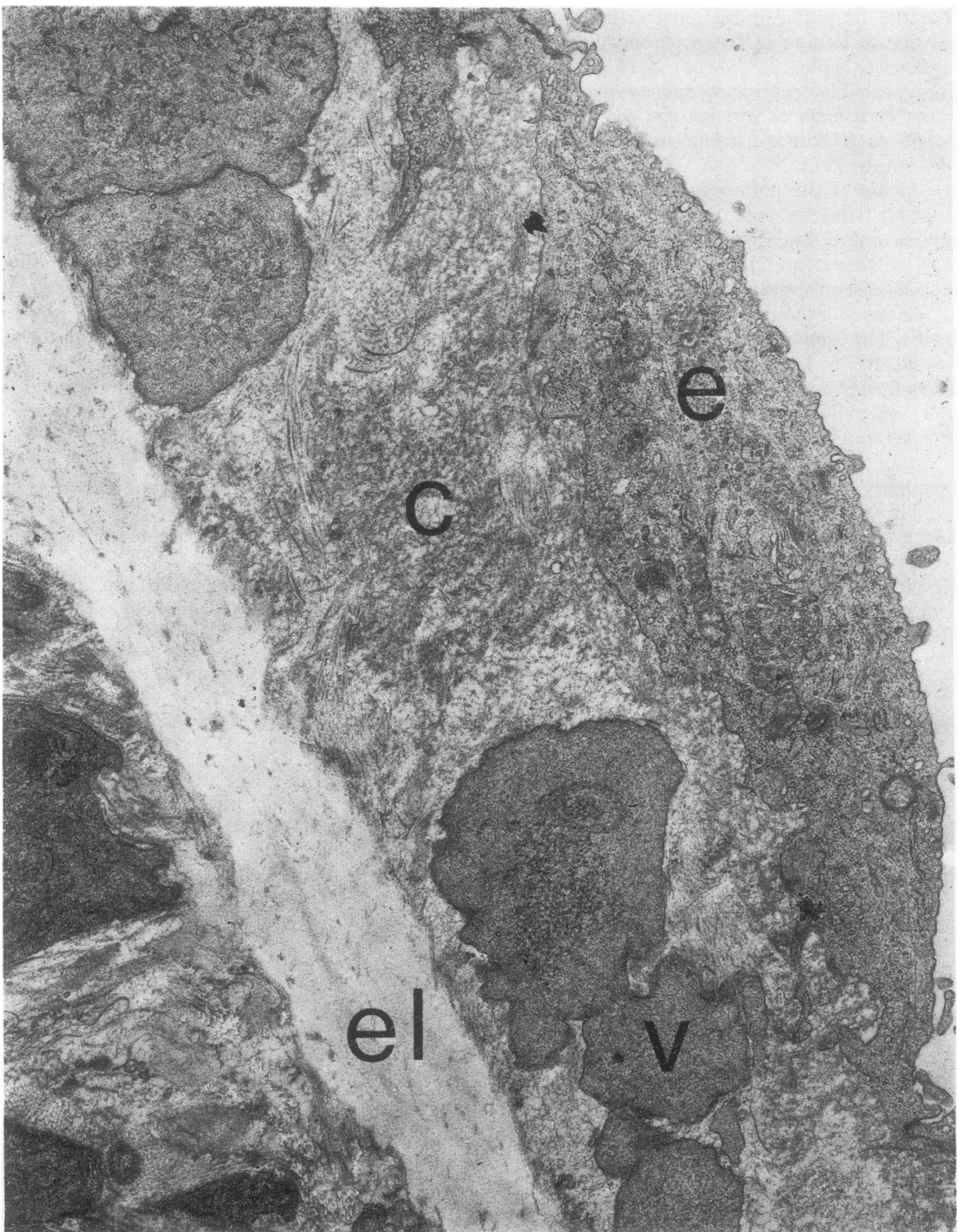

Fig. 3 Pulmonary trunk after one week of hypoxia. Numerous evaginations of smooth muscle (v) are present in the subendothelial connective tissue (c) between the endothelium (e) and the internal elastic lamina (el). These evaginations have the same density as the parent smooth muscle cells. Electron micrograph $\times 12500$. 


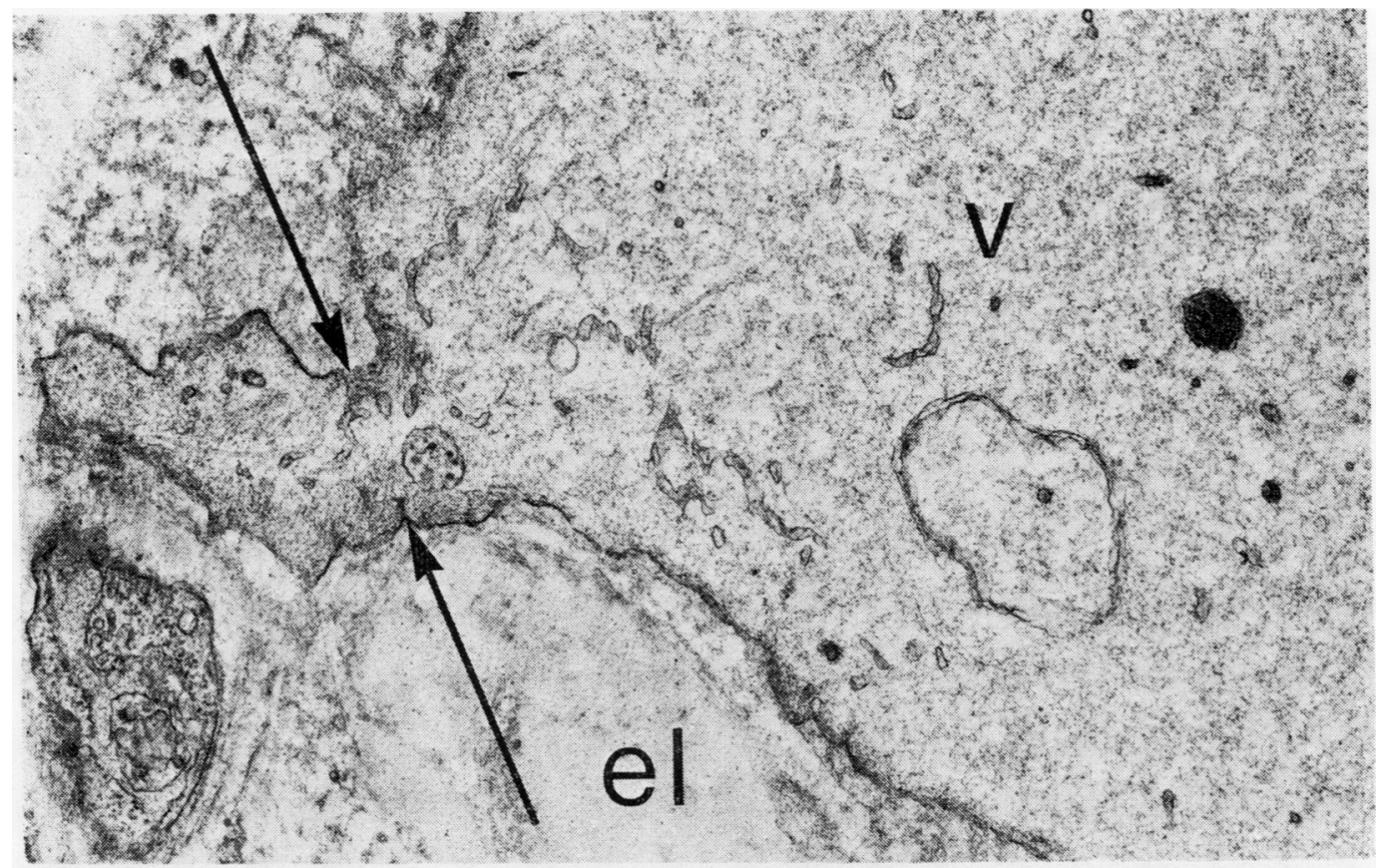

Fig. 4 An evagination of smooth muscle (v) caught in the process of extrusion through a gap (arrows) in the internal elastic lamina (el). The direction of the intima is to the right of the picture. Note that the evagination lacks myofilaments and mitochondria, and there is disruption of the membranes of the endoplasmic reticulum. Electron micrograph $\times 25000$.

structures were unrecognisable as muscle except for their occasional connection with the media through a gap in the internal elastic lamina (Fig. 6). In other instances, the muscular evaginations were almost totally empty and consisted merely of large cyst-like spaces in the intima resembling vacuoles (Fig. 7). Several smooth muscle cells showed small lateral protuberances, which extended towards the intima but which did not traverse the elastic lamina. Presumably these evaginations formed in places where there was no gap in the elastic lamina for them to pass through (Fig. 8).

The muscle cells in the remainder of the media showed little abnormality except for the occasional occurrence of small regions of apparently degenerate smooth muscle (Fig. 9). It was not clear whether these represented evaginations of smooth muscle taking place within the media.

\section{Exposed to four weeks of hypoxia}

After exposure to hypoxia for four weeks evaginations of smooth muscle were still present but less common than after one week of exposure. The subendothelial connective tissue was thicker than normal and contained irregular amorphous focal deposits resembling ground substance. These deposits were usually closely associated with downward extensions of the endothelial cells. The smooth muscle cells of the media were markedly hypertrophic. They were twice the thickness of smooth muscle cells in the controls and contained an abundance of myofilaments orientated mutually parallel in the longitudinal axis of the cell. Muscle cells also contained many mitochondria and membranes of rough endoplasmic reticulum, usually situated in small groups within spaces between the myofilaments. The muscle cells appeared to be secreting a finely fibrillary material at their periphery but the nature of this material was unclear.

\section{Discussion}

Our study shows that on acute exposure to hypoxia ultrastructural changes develop in the pulmonary trunk. These comprise evaginations of smooth muscle cells, some of which insinuate through gaps in the internal elastic lamina to reach the intima. Usually the cytoplasm of these protuberances is devoid of myofilaments and largely free of cell organelles. In some instances it is so electron-lucent as to look more like a 


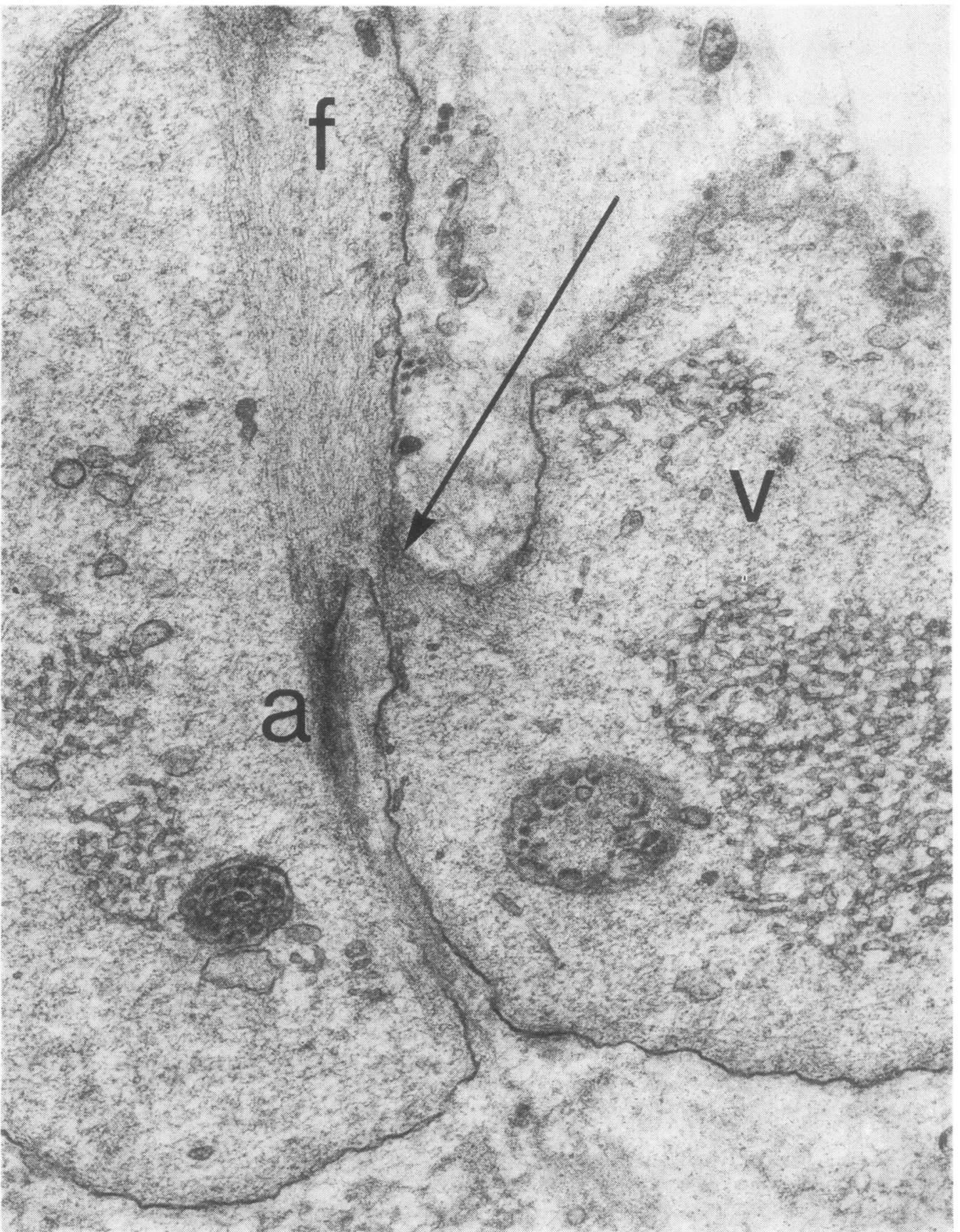

Fig. 5 An evagination (v) of smooth muscle within the subendothelial connective tissue. There is reduced density of the cytoplasm with loss of mitochondria and disruption of endoplasmic reticulum. The parent muscle cells contain myofilaments $(f)$ running to a dense attachment point $(a)$. Note the narrow isthmus of tissue (arrow) by which the evagination is connected to the parent muscle cell. Electron micrograph $\times 50000$. 


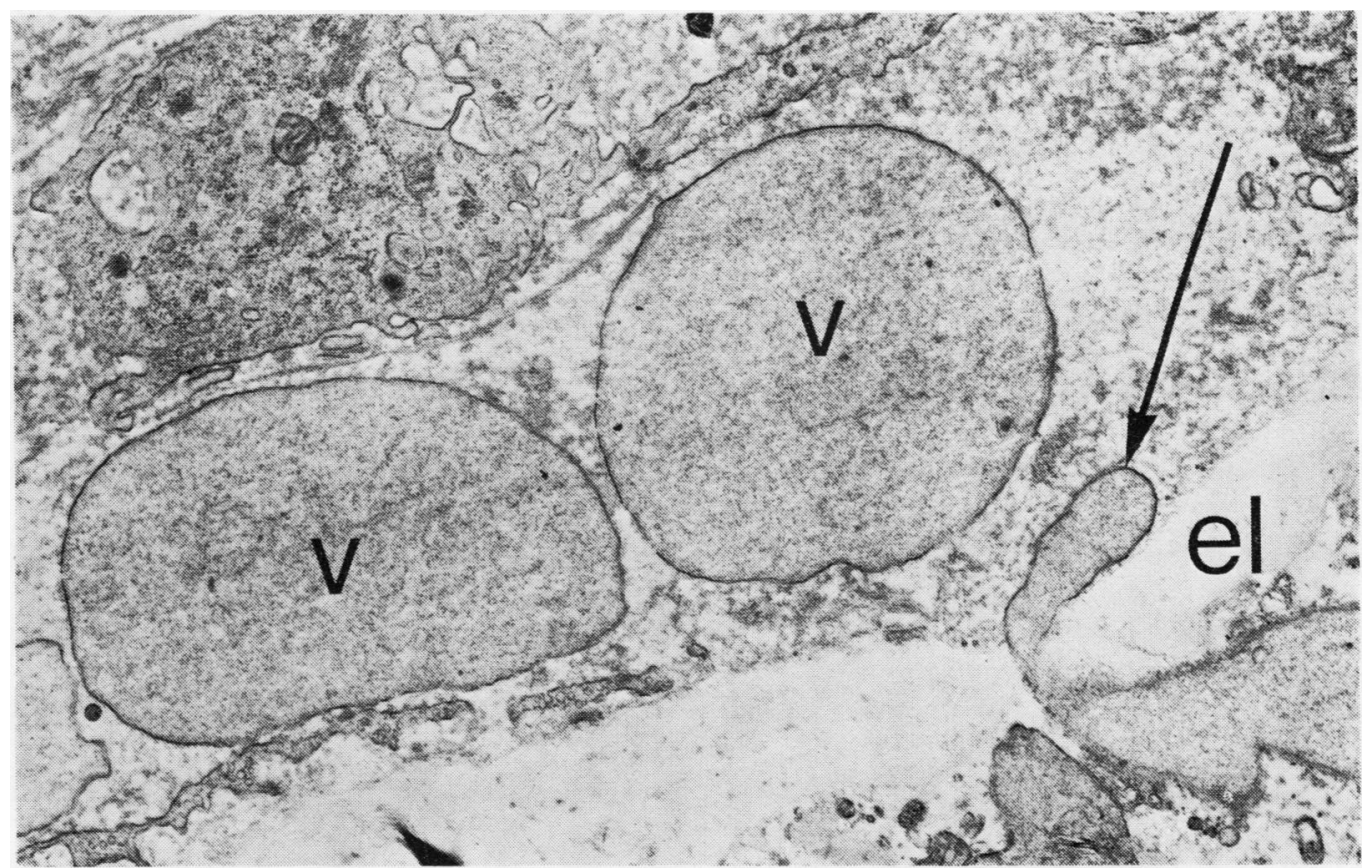

Fig. 6 Smooth muscle evaginations $(v)$ in which there is total loss of cellular detail. These structures bear no resemblance to the muscle from which they are derived. In the lower right-hand corner, a small muscular evagination (arrow) has extruded through a gap in the internal elastic lamina (el). Electron micrograph $\times 12500$.

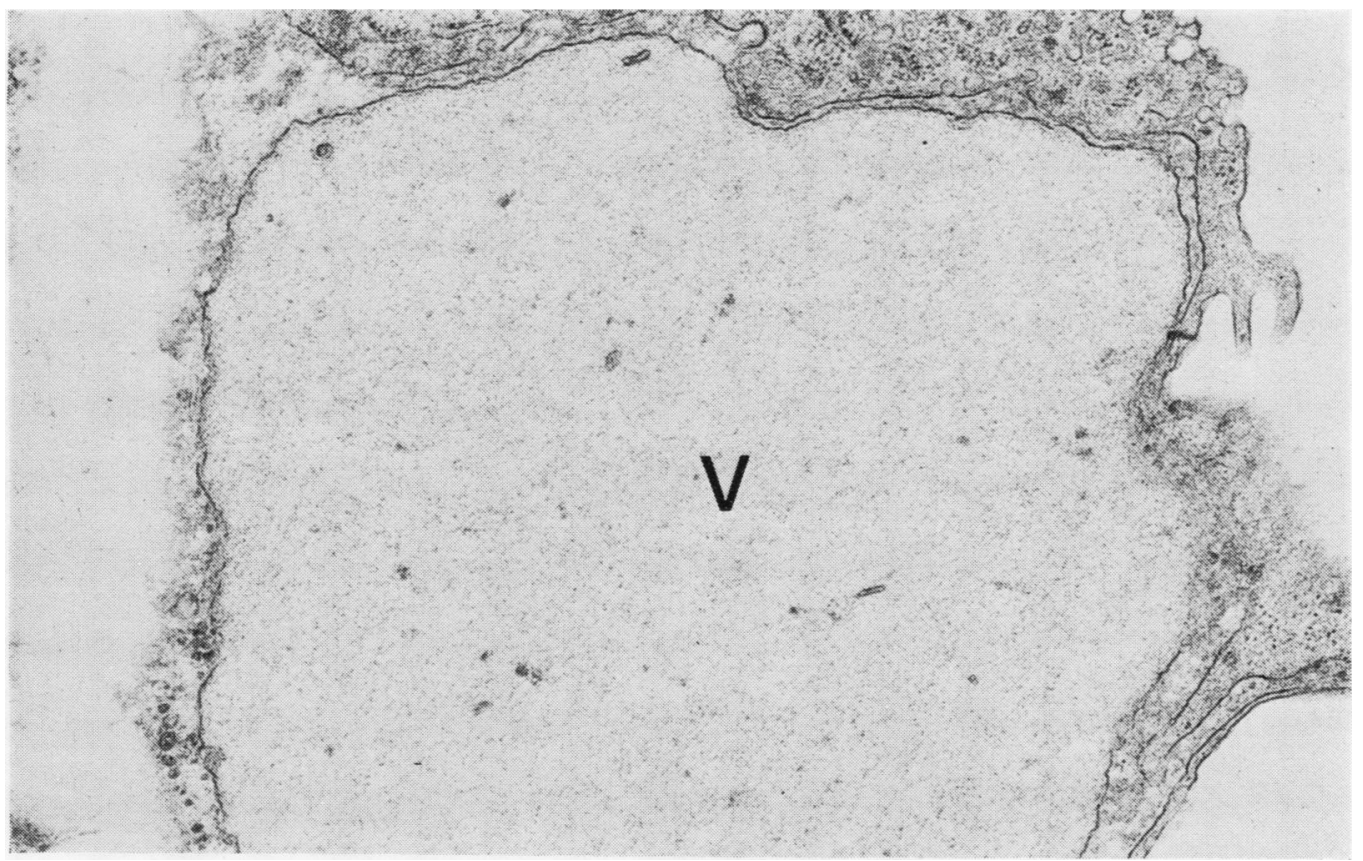

Fig. 7 An evagination of smooth muscle (v), in which disruption of the cytoplasm is so extreme that it consists merely of a cystic space resembling a vacuole. Electron micrograph $\times 37500$. 


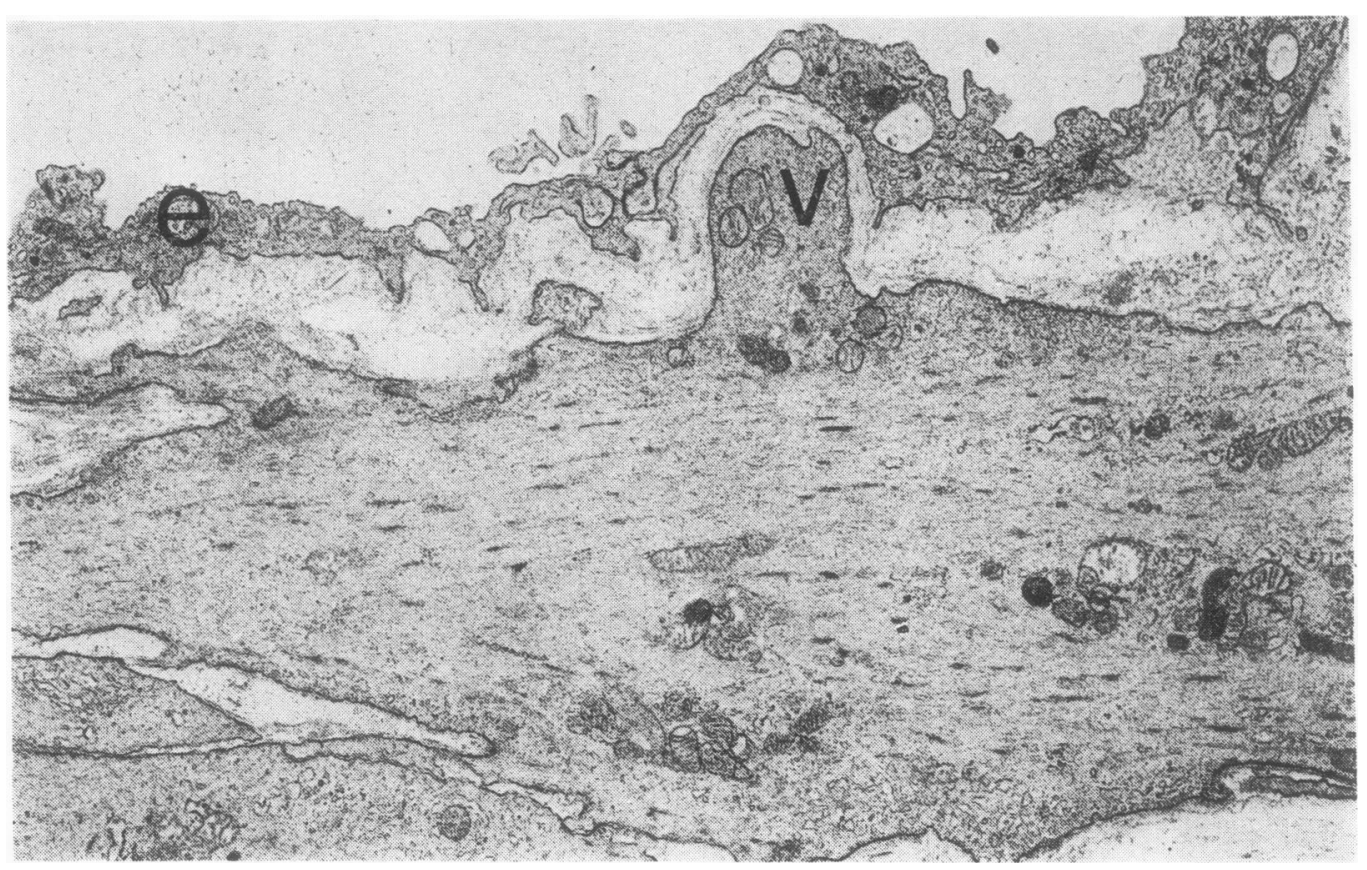

Fig. 8 An evagination of smooth muscle (v) extending towards the endothelium (e). It has not traversed the internal elastic lamina. Electron micrograph $\times 7500$.

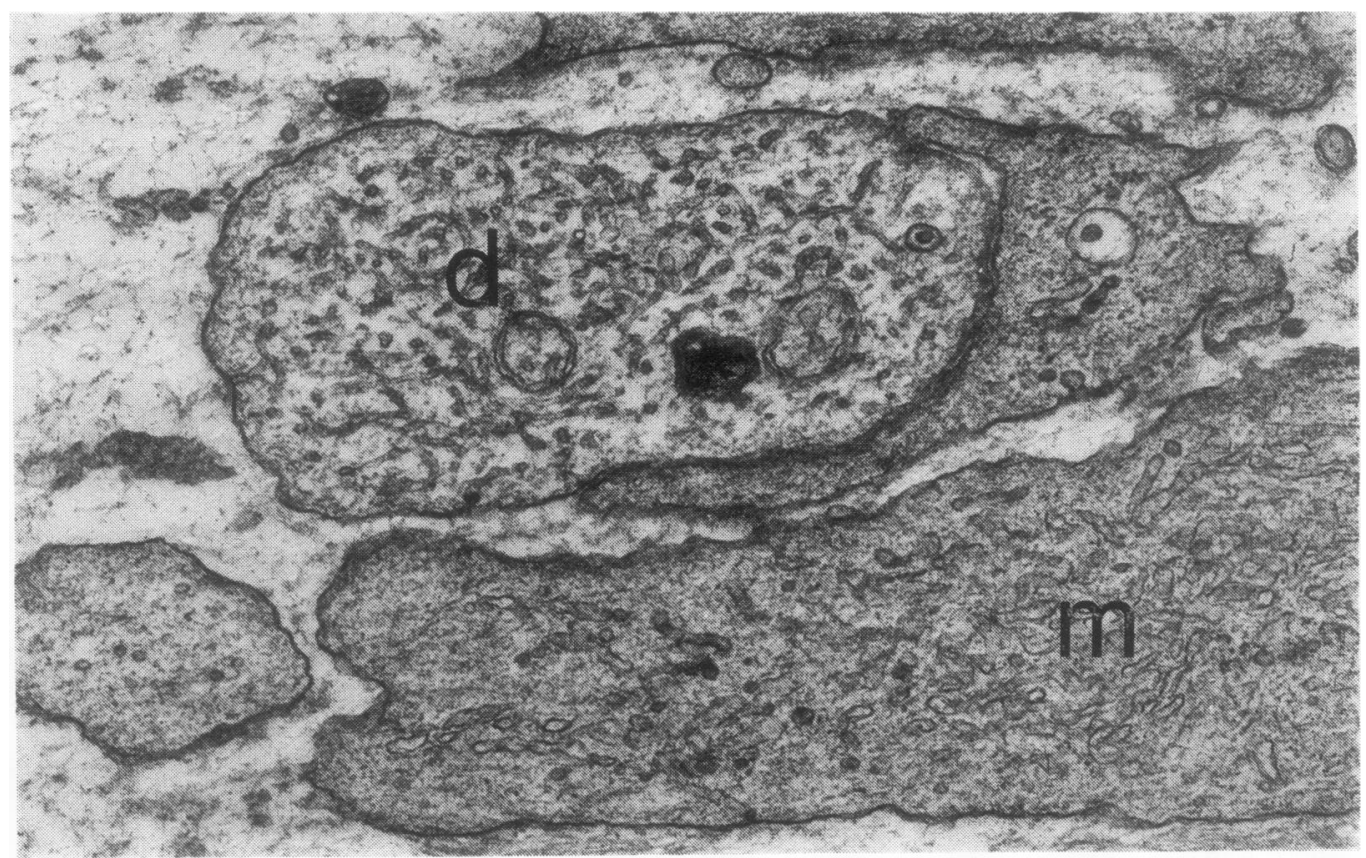

Fig. 9 An area of pale degenerating smooth muscle $(d)$ next to normal healthy smooth muscle $(m)$. This may represent a muscle evagination within the media. Electron micrograph $\times 37500$. 
cyst in the intima than a derivative of muscle. We took great care to fix the pulmonary trunk in distension because elsewhere (Smith et al., 1978) we have demonstrated that muscular evaginations can occur in normal pulmonary blood vessels as an artefact brought about by collapse of lung tissue and they can be avoided by distending the lung. Hence in the present investigation, in which the pulmonary trunk was distended with fixative until it assumed the same dimensions that it had in vivo, the evaginations are interpreted as indicating contraction of the muscle cells able to overcome the distending force. We interpret the evaginations as evidence of constriction of muscle cells in the media of the pulmonary trunk in response to hypoxia.

It has been known for over a decade that, when smooth muscle cells contract, bulbous protuberances arise from their surface. Thus in 1965 Lane commented on alterations in the cytological detail of intestinal smooth muscle cells in various stages of contraction. Fay and Delise (1973) studied the morphological alterations in a contracting single muscle cell from the stomach of Bufo marinus by means of scanning and transmission electron microscopy and by cinematography. They found that in the relaxed cell the surface was smooth and the cytoplasmic myofilaments were orientated longitudinally. On contraction, however, the cell surface became covered in bulbous and mound-like protuberances. The myofilaments in the contracted cells became densely packed together and assumed an apparently random distribution in the cell. In the overlying plasma membrane of the body of the cell were abundant dense bodies (attachment points). It is likely that these plaques represent the points where the actin filaments anchor to the sarcolemma (Kelly and Rice, 1969) (Fig. 10). The bulbous evaginations were largely free of myofilaments, and their covering of plasma membrane was relatively clear of the dense bodies referred to above. Fay and Delise (1973) believe that, when the myofilaments contract, they exert an inwardly directing force, which produces invaginations of the muscle cytoplasm at the points where dense bodies are situated. At the same time cytoplasm free of myofilaments is squeezed out under areas of plasma membrane free of dense bodies (Fig. 10). Since such evaginations are a normal feature of contraction of smooth muscle one would anticipate finding them in electron micrographs of smooth muscle cells which had been subjected to sustained contraction. These conditions were realised in the present investigation in which the pulmonary trunk was

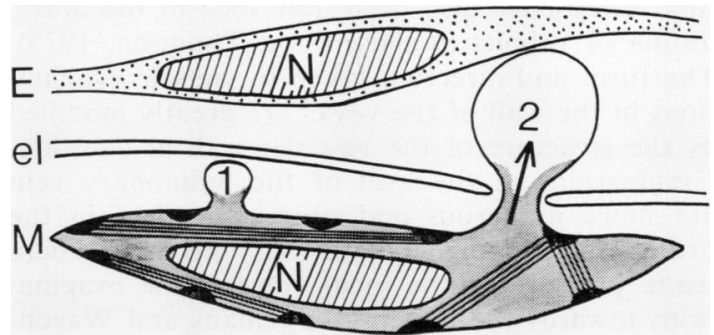

Fig. 10 Diagrammatic representation of the inner part of the wall of the pulmonary trunk consisting of an endothelial cell $(E)$ with its nucleus $(N)$, the internal elastic lamina (el), and a smooth muscle cell $(M)$. The myofilaments are represented by sets of parallel lines fixed to the dark attachment points around the periphery of the cell. The muscle is in a contracted state, and the inwardly directed pull of the myofilaments squeezes out the cytoplasm between adjacent attachment points in the form of bulbous evaginations. Where the overlying elastic lamina is intact, the evaginations are contained within a small space (1). Where there is a gap in the elastic lamina the evaginations can pass through and expand in the loose subendothelial connective tissue (2). Here they form large islands of pale cytoplasm, such as one sees after exposure to hypoxia for one week.

subjected to the stimulus of hypoxia, a most potent constrictor of pulmonary vascular smooth muscle (Harris and Heath, 1977). Muscular evaginations have also been found in the pulmonary vasculature when the stimulus for vasoconstriction was the pyrrolizidine alkaloid fulvine (Dingemans and Wagenvoort, 1976). However, the evaginations in the pulmonary trunk differ in some respects from those produced by Fay and Delise (1973) in isolated muscle cells or by Dingemans and Wagenvoort (1976) in small pulmonary arteries and veins. They were significantly larger and sometimes contained endoplasmic reticulum and remnants of myofilaments. This suggests that part of an entire smooth muscle cell passes through a gap in the internal elastic lamina rather than merely peripheral clear cytoplasm between adjacent attachment points. It is conceivable, therefore, that in the pulmonary trunk muscular evagination is aided or superseded by active migration of smooth muscle cells into the intima followed by their degeneration.

It seems likely that evaginations of muscle cells are to be found in any type of blood vessel when it constricts. Thus, while we have reported the occurrence of evaginations in the pulmonary trunk, Dingemans and Wagenvoort (1976) found them in the small pulmonary arteries and veins of 
rats. They have also been described in the aortic intima of rabbits (Kjeldsen and Thomsen, 1975). The form and direction taken by muscle evaginations in the wall of the vessel are greatly modified by the structure of the vascular wall in question. Evaginations in the wall of the pulmonary vein are more numerous and prominent than in the firmer, more compact pulmonary arteries, where tissue pressure in the media directs the evaginations towards the lumen (Dingemans and Wagenvoort, 1976). The same inward direction of the protuberances was found in the present study, and Kjeldsen and Thomsen (1975) found the same tendency in the aorta so that the vesicles appeared within the intima. The relation of these evaginations of muscle to elastic laminae is of interest since the extrusions of muscle appear to insinuate through gaps in the laminae to expand bulbously in the intima. The incomplete elastic laminae of arterioles and veins offer the best opportunities for the formation of such extrusions, but fenestrations of laminae in any class of vessel make the process possible. Even allowing for the fact that the evaginations are usually largely devoid of myofilaments and organelles, they appear to become more electron-lucent in their intimal situa- tion. It is conceivable that this abnormal lucency $\stackrel{\vec{F}}{\rightarrow}$ could proceed to degenerative changes in these portions of cytoplasm connected to the body of the cell only by a narrow isthmus.

The extrusion of the cytoplasm of smooth $\stackrel{\mathbb{D}}{2}$ muscle cells through gaps in elastic laminae into \& the intima, and the fact that this may occur in $\vec{\circ}$ muscular pulmonary arteries reawakens interest in the interpretation of certain aspects of 'hypoxic $\vec{\omega}$ hypertensive pulmonary vascular disease' (Hasle- $\stackrel{\circ}{\circ}$ ton et al., 1968). In this condition, which may $\underset{x}{\vec{x}}$ complicate chronic bronchitis and emphysema and $\underset{\omega}{\omega}$ other diseases leading to chronic alveolar hypoxia, a layer of smooth muscle appears in the intima $\dot{\omega}$ (Fig. 11). This has been interpreted in the past as due solely to a newly formed layer of longitudinal muscle, and indeed nuclei can be seen in some of $\vec{T}$ these muscle fibres cut in transverse section. How- $\frac{\mathbb{D}}{O}$ ever, one wonders whether some of this intimal muscularisation might not be due to the phenomenon of muscular evagination that we describe in this paper. Certainly, if some of these nodules of muscle in the intima were due to extensions of cell cytoplasm which subsequently underwent degeneration, it would explain the hitherto unexplained sequel of intimal fibrosis and elastosis

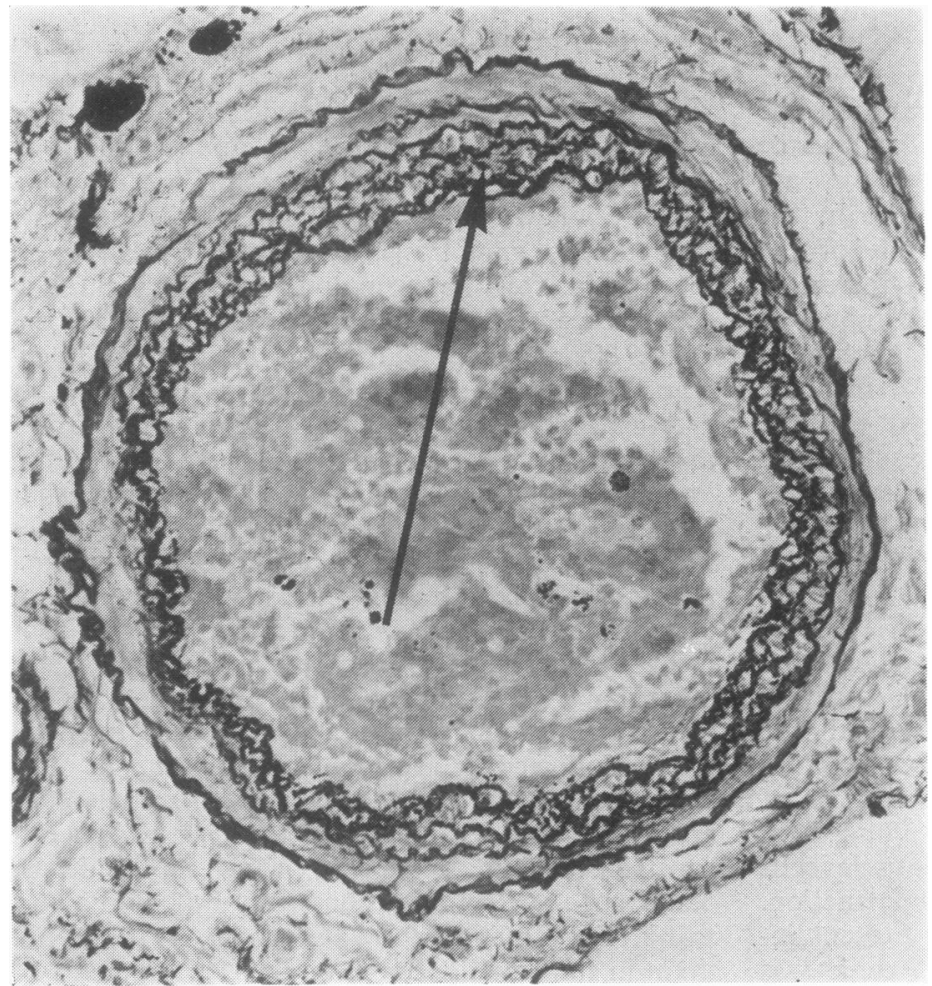

Fig. 11 Transverse section of a muscular pulmonary artery from a man who died with centrilobular emphysema. The intima of the vessel consists of a ring of black anastomosing elastic fibres with smooth muscle cells in its interstices (arrow). The muscle appears to be orientated longitudinally, but some of these cells may be evaginations of smooth muscle from the media. Elastic van Gieson $\times 300$. 


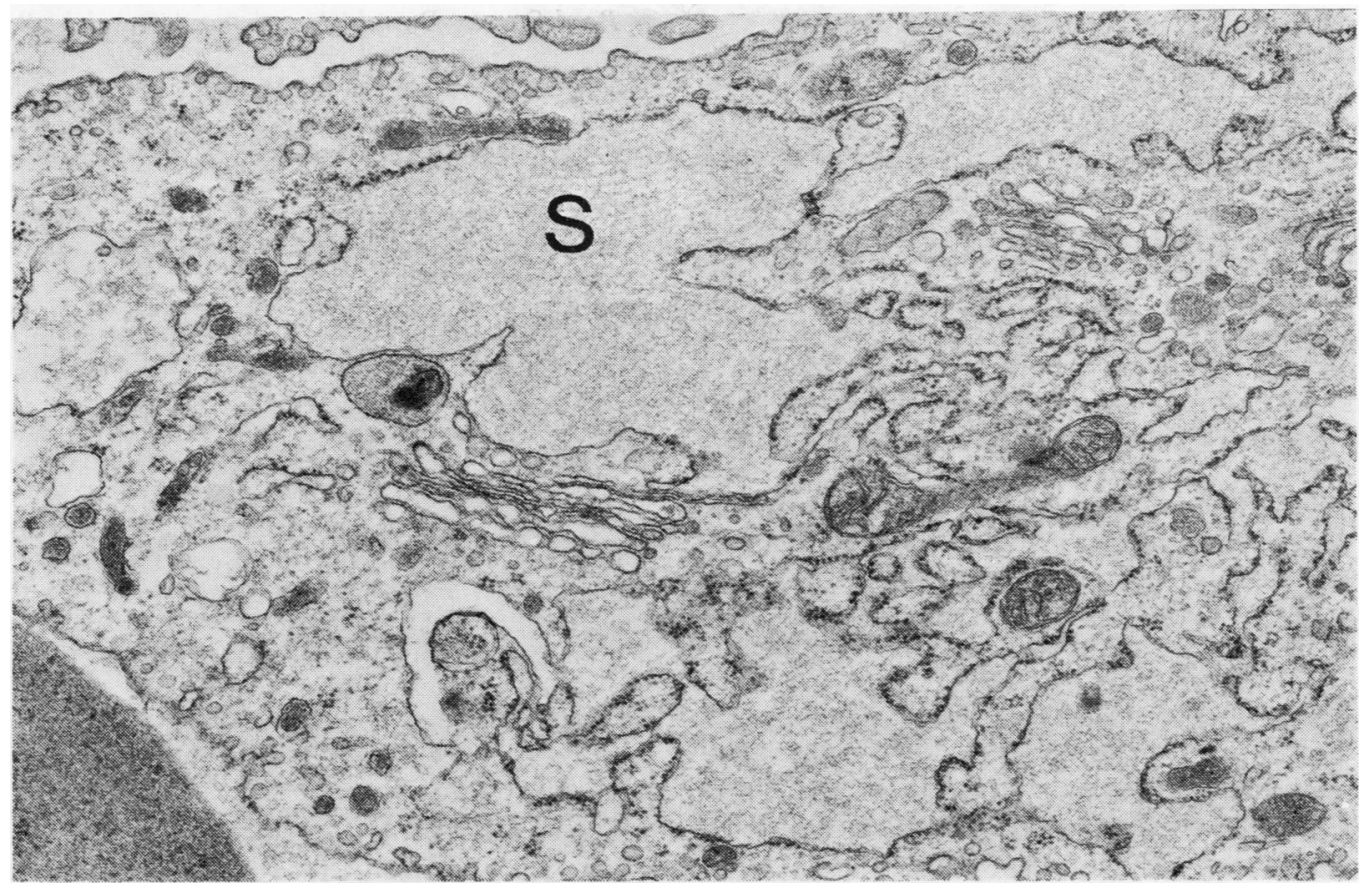

Fig. 12 An endothelial cell from a muscular pulmonary artery of a rat exposed to a barometric pressure of $380 \mathrm{mmHg}$ for two weeks. There is dilatation of the rough endoplasmic reticulum to produce cyst-like spaces (s). The spaces contain a pale granular material and are lined by a single membrane bearing ribosomes. Electron micrograph $\times 25000$.

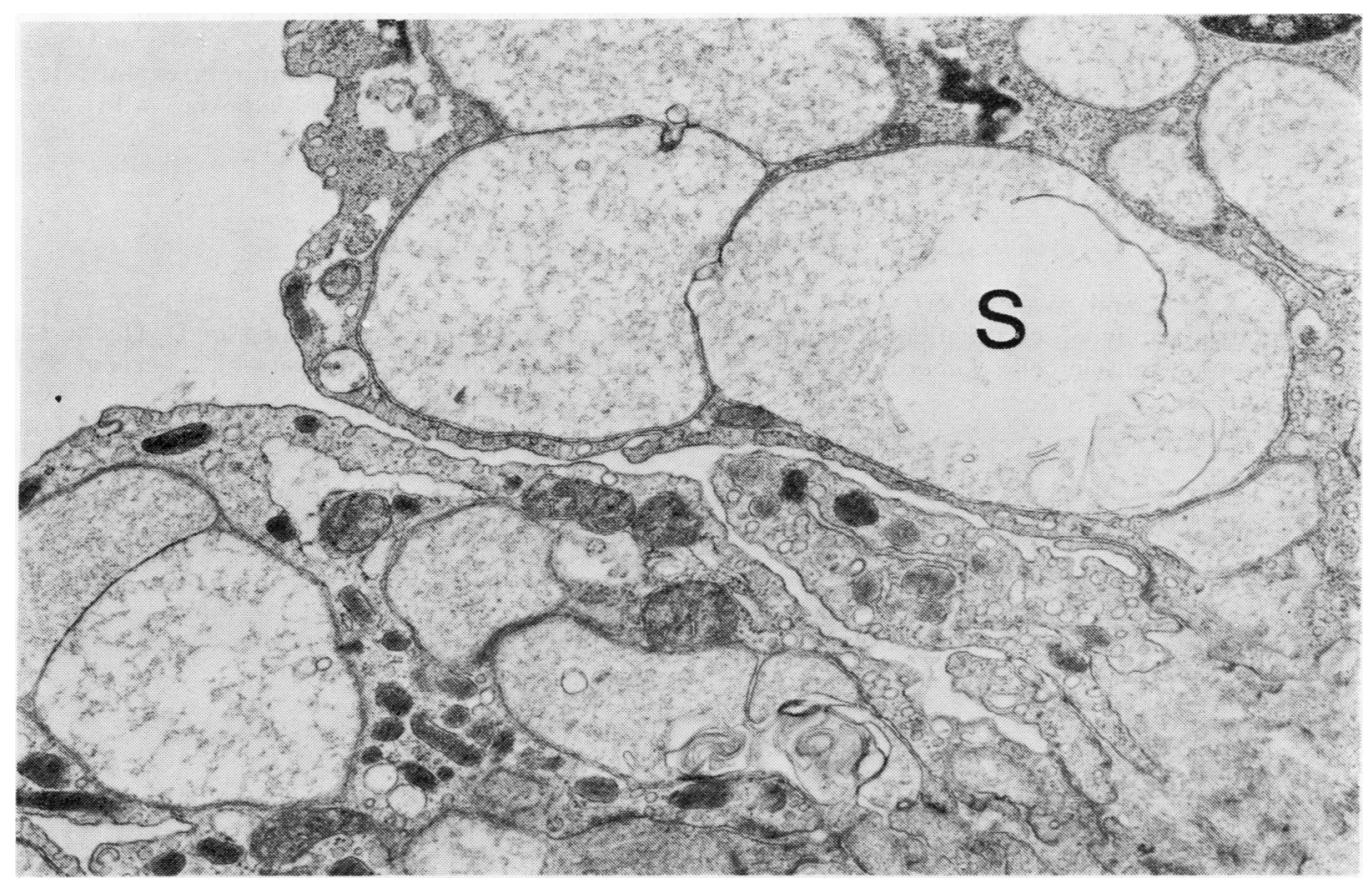

Fig. 13 Two endothelial cells from a pulmonary vein of a rat exposed to a barometric pressure of $380 \mathrm{mmHg}$ for three weeks. The cells contain large, almost empty cystic spaces (s). In contrast to the spaces shown in Fig. 12, these are lined by a double membrane, which does not bear ribosomes. They are likely to be evaginations of smooth muscle. The space between the double membranes probably represents the interstitial space between endothelium and muscular evaginations. Electron micrograph $\times 18750$. 
to replace the muscle tissue in hypoxic hypertensive pulmonary vascular disease.

In a recent study of the ultrastructure of hypoxic hypertensive pulmonary vascular disease (Smith and Heath, 1977), we observed cystic changes in the intima of pulmonary arterioles and veins. Some of these cysts were produced by cisternal dilatation of the rough endoplasmic reticulum in endothelial cells (Fig. 12). Others, however, were lined by a double membrane (Fig. 13), and it seems likely that they were produced by evagination of muscle cells of the underlying media such as we have described above. Hence, it seems likely to us that in states of chronic hypoxia evaginations of smooth muscle cells occur throughout the entire pulmonary vasculature to include pulmonary trunk, muscular pulmonary arteries, pulmonary arterioles, and pulmonary veins.

Finally, it is not without interest to note that in this study we have ultrastructural evidence of contraction of smooth muscle cells in the innermost portions of the pulmonary trunk in animals subjected to severe and sustained hypoxia. Mast cells are not in proximity to these muscle cells and hence cannot be given the rôle of intermediary agent in bringing about their contraction. This tends to support the view that mast cells are not involved in hypoxic vasoconstriction of the terminal portion of the pulmonary arterial tree (Williams et al., 1977).

This work was carried out with the aid of a grant from the British Heart Foundation.

\section{References}

Dingemans, K. P., and Wagenvoort, C. A. (1976). Ultrastructural study of contraction of pulmonary vascular smooth muscle cells. Laboratory Investigation, 35, 205-212.
Fay, F. S., and Delise, C. M. (1973). Contraction of $\frac{\overline{\bar{N}}}{7}$ isolated smooth muscle cells-structural changes. Proceedings of the National Academy of Sciences, USA, 70, 641-645.

Harris, P., and Heath, D. (1977). The Human Pul- $\stackrel{\mathbb{D}}{\Omega}$ monary Circulation. 2nd edition. Churchill Livingstone, Edinburgh.

Hasleton, P. S., Heath, D., and Brewer, D. B. (1968). $\vec{\circ}$ Hypertensive pulmonary vascular disease in states $\overrightarrow{\vec{H}}$ of chronic hypoxia. Journal of Pathology and $\vec{\omega}$ Bacteriology, 95, 431-440.

Kelly, R. E., and Rice, R. V. (1969). Ultrastructural $\underset{x}{\vec{x}}$ studies on the contractile mechanism of smooth muscle. Journal of Cell Biology, 42, 683-694.

Kjeldsen, K., and Thomsen, H. K. (1975). The effect $\dot{\omega}$ of hypoxia on the fine structure of the aortic intima $\overrightarrow{ }$ in rabbits. Laboratory Investigation, 33, 533-543.

Lane, B. P. (1965). Alterations in the cytologic detail of intestinal smooth muscle cells in various stages $T$ of contraction. Journal of Cell Biology, 27, 199-213. 음

Smith, P. (1977). A comparison of the orientation of elastin fibers in the elastic laminae of the pulmonary trunk and aorta of rabbits using the scanning $\vec{\varphi}$ electron microscope. Laboratory Investigation, 35, 525-529.

Smith, P., and Heath, D. (1977). Ultrastructure of hypoxic hypertensive pulmonary vascular disease. Journal of Pathology, 121, 93-100.

Smith, P., Heath, D., and Mooi, W. (1978). Observations on some ultrastructural features of normal pulmonary blood vessels in collapsed and distended lungs. Journal of Anatomy, (in press).

Williams, A., Heath, D., Kay, J. M., and Smith, P. 3 (1977). Lung mast cells in rats exposed to acute $\supset$ hypoxia, and chronic hypoxia with recovery. Thorax, 32, 287-295.

Requests for reprints to: Professor D. Heath, Department of Pathology, University of Liverpool, PO Box 147, Liverpool L69 3BX. 